\title{
A real-time virtual delivery system for photon radiotherapy delivery monitoring
}

\author{
Feng Shi, Xuejun Gu, Yan Jiang Graves, Steve Jiang, Xun Jia \\ Division of Medical Physics and Engineering, Department of Radiation Oncology University of Texas Southwestern Medical Center, \\ Dallas, TX, USA.
}

Received March 19, 2014; Published Online April 08, 2014

\begin{abstract}
[Presented at the Young Investigator's Symposium at the 2014 Annual Meeting of Southwest Chapter of American Association of Physicists in Medicine (AAPM) in San Antonio, Texas, USA]
\end{abstract}

\section{Conference Proceeding}

\begin{abstract}
Purpose: Treatment delivery monitoring is important for radiotherapy, which enables catching dosimetric error at the earliest possible opportunity. This project develops a virtual delivery system to monitor the dose delivery process of photon radiotherapy in real-time using GPU-based Monte Carlo (MC) method.
\end{abstract}

Methods: The simulation process consists of 3 parallel CPU threads. A thread T1 is responsible for communication with a linac, which acquires a set of linac status parameters, e.g. gantry angles, MLC configurations, and beam MUs every 20 $\mathrm{ms}$. Since linac vendors currently do not offer interface to acquire data in real time, we mimic this process by fetching information from a linac dynalog file at the set frequency. Instantaneous beam fluence map (FM) is calculated based. A FM buffer is also created in T1 and the instantaneous FM is accumulated to it. This process continues, until a ready signal is received from thread T2 on which an in-house developed MC dose engine executes on GPU. At that moment, the accumulated FM is transferred to T2 for dose calculations, and the FM buffer in T1 is cleared. Once the dose calculation finishes, the resulting $3 \mathrm{D}$ dose distribution is directed to thread T3, which displays it in three orthogonal planes in color wash overlaid on the CT image. This process continues to monitor the $3 \mathrm{D}$ dose distribution in real-time.

Presenting author: Feng Shi; Division of Medical Physics and Engineering, Department of Radiation Oncology University of Texas Southwestern Medical Center, Dallas, TX, USA.

Cite this article as:

Shi F, Gu X, Graves YJ, Jiang S, Jia X. A real-time virtual delivery system for photon radiotherapy delivery monitoring. Int J Cancer Ther Oncol 2014; 2(2):020222.

DOI: $10.14319 /$ ijcto.0202.22
Results: An IMRT and a VMAT cases used in our patient-specific QA are studied. Maximum dose differences between our system and treatment planning system are $0.98 \%$ and $1.58 \%$ for the IMRT and VMAT cases, respectively. The update frequency is $>10 \mathrm{~Hz}$ and the relative uncertainty level is $2 \%$.

Conclusion: By embedding a GPU-based MC code in a novel data/work flow, it is possible to achieve real-time MC dose calculations to monitor delivery process.

Key Results: An IMRT and a VMAT cases are studied on a water phantom, where real patient cases generated for patient-specific QA purpose. The plans are delivered on a Varian TrueBeam linac and the dynalog files are extracted. Figure 1 shows the main software interface of our system at various moment of the delivery process.

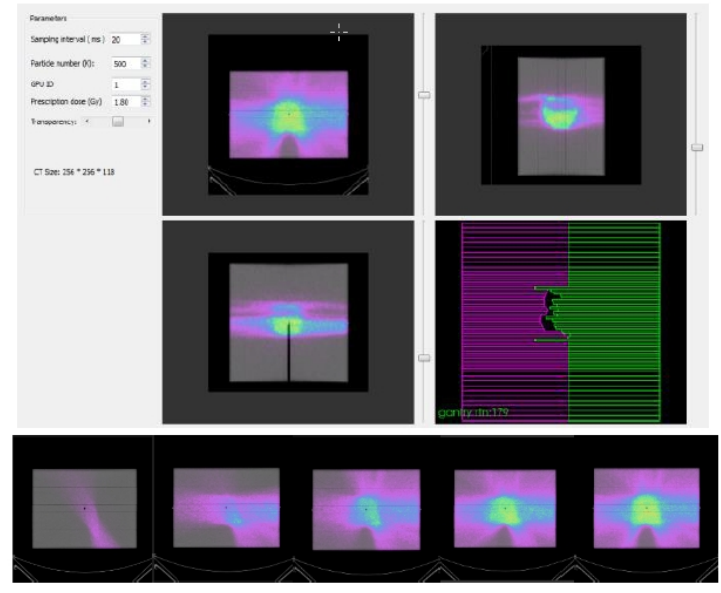

FIG. 1: Main software interface (top). Once slice of 3D dose at different time points (bottom). 
We first validate the accuracy of our dose calculations. As such, the simulated dose as a function of time is recorded. The IMRT and the VMAT plan are also split into many plans corresponding to different time point and doses are calculated in our Treatment Planning System, Eclipse. These doses at the chamber point (air cavity of the phantom) are compared and the results are shown in Figure 2. We can see that maximum dose difference between the simulation and the TPS are $0.98 \%$ and $1.58 \%$ for the IMRT and the VMAT cases, respectively.
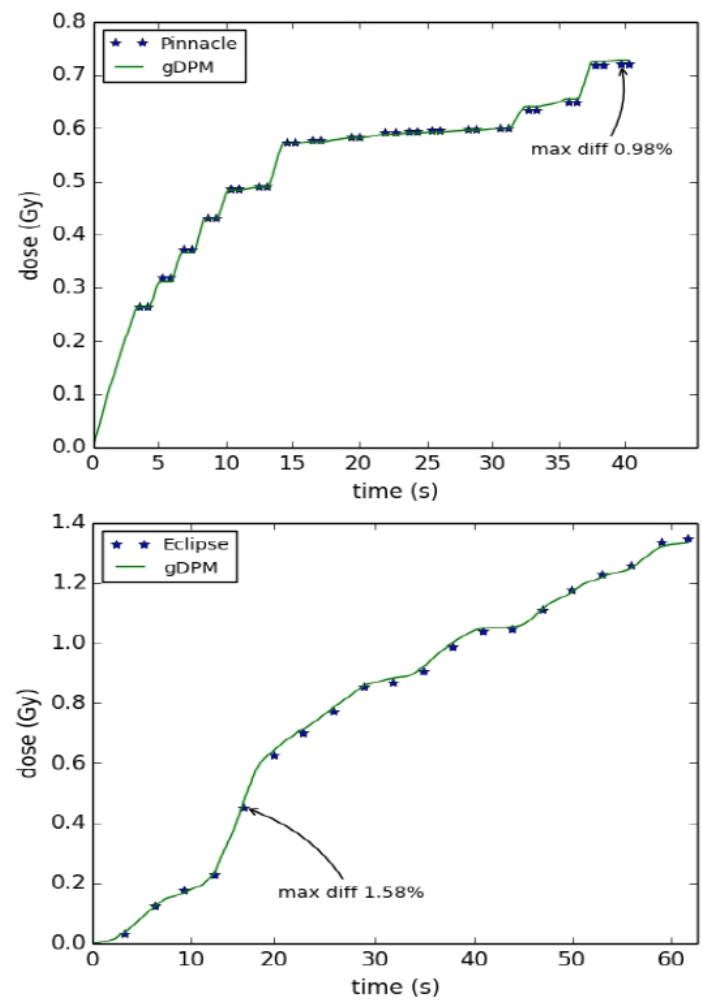

FIG. 2: Comparison of dose of the simulation and TPS for the IMRT (top) and VMAT (bottom) cases.
The MC code gDPM is repeated launched to update 3D dose distribution in real-time. The average computing time per gDPM execution $\bar{t}$ depends on the number of particles simulated each time $=\alpha A$, which is further proportional to the jaw openning area $A$. The larger $\alpha$ is, the more particles are simulated, leading to increased execution time. Meanwhile, the parameter $\alpha$ governs the particle fluence from the source impinging the patient during MC simulations. Hence the uncertainty will be reduced by using $\alpha$ large values. It is hence less meaningful to report the absolute update frequency without mentioning the uncertainty level, as one can always improve the temporal resolution at the price of sacrificing the uncertainty level. Rather, it is interesting to observe the trade-off between the two quantities. As such, we run calculations with a set of values and obtain the corresponding $\bar{t}$ and the average relative uncertainty $\left\langle\sigma_{\mathrm{D}} / \mathrm{D}_{\mathrm{p}}\right\rangle$ under each $\alpha$ value. Here $\sigma_{D}$ is the absolute uncertainty of dose in a voxel and $D_{p}$ is the prescription dose, and $\langle$.$\rangle indicates an$ average over voxels within the PTV region. Curves in Figure 3 clearly demonstrate the tradeoff of the two quantities. Particularly, we can achieve $\sim 1 \%$ uncertainty level at an update frequency of $10 \mathrm{~Hz}$.

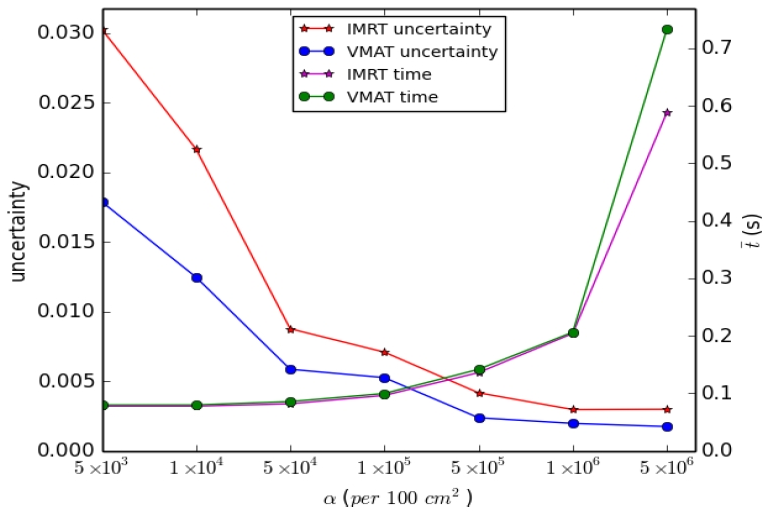

FIG. 3: Comparison of uncertainty and average gDPM execution time. 